Check for updates

Cite this: Phys. Chem. Chem. Phys., 2018, 20, 18718

Received 29th March 2018, Accepted 10th June 2018

DOI: $10.1039 / c 8 c p 02027 b$

rsc.li/pccp

\title{
Understanding the non-covalent interaction mediated modulations on the electronic structure of quasi-zero-dimensional graphene nanoflakes $\dagger$
}

\begin{abstract}
Amrit Sarmah (D) $\star^{\mathrm{ab}}$ and Pavel Hobza (D) ${ }^{\mathrm{ab}}$
In recent years, magnetic or electric field induced modulations on the electronic environment of single molecular systems are common practice. In this particular study, we have instigated the possibility of controlling the electronic and spin-dependent properties of hydrogen-terminated graphene fragments, so-called graphene nanoflakes (GNF), using weak non-covalent interactions as the external stimuli. The topological frustration in the graphene fragment appreciated the compelling electronic behavior of the system. This leads to some unorthodox spin-distribution in the system and it is possible to synchronize this electronic perturbation switching through a non-covalent interaction. These findings institute a new avenue for sculpting such donor-acceptor composites as self-regulated spintronic devices in next generation electronics.
\end{abstract}

\section{Introduction}

Low dimensional atomically precise carbon-based magnetism ${ }^{1}$ consisting of a $2 \mathrm{D}$ graphene backbone is anticipated to be the most suitable building-block for the fabrication of nextgeneration electronic and spintronics devices. ${ }^{2}$ The higher Curie temperature and relatively large spin correlation length compared to those of conventional magnets are primarily responsible for the exquisite features in the carbon-based magnetic nanostructures. ${ }^{3}$ In a recent technological breakthrough, the laboratory synthesized true two-dimensional (2D) graphene crystals, which generated massive excitement among the scientific community across the globe. ${ }^{4,5}$ Subsequent sophisticated investigations of this system revealed different, extraordinary electronic properties such as the room-temperature quantum hall effect, ${ }^{6}$ half-metallicity, ${ }^{7}$ massless quasi-particles, ${ }^{8}$ high mobility and coherence, ${ }^{4}$ etc. Although developing a precise understanding of these properties accounts for some fundamental perspectives, these have considerable implications on high-end technological developments as well. On the lower side, the inherent

\footnotetext{
${ }^{a}$ Institute of Organic Chemistry and Biochemistry of the Czech Academy of Sciences, Flemingovo nam. 2, CZ-16610 Prague 6, Czech Republic. E-mail: amrit.sarmah@uochb.cas.cz

${ }^{b}$ Department of Physical Chemistry, Palacký University, CZ-77146 Olomouc, Czech Republic

$\dagger$ Electronic supplementary information (ESI) available: Pictorial representations of HATCN structure; computed 2D charge density distribution contour maps; computed DOS plots; computed total magnetization values for the five GNFHATCN composite systems; computed differential charge density (DCD) distribution maps. See DOI: $10.1039 / \mathrm{c} 8 \mathrm{cp} 02027 \mathrm{~b}$
}

zero bandgap and semi-metal characteristics are the severe impediments that restrain the feasibility of carbon-based electronics. Certain promising topological modifications to the $2 \mathrm{D}$ graphene crystal lattice to engineer a small band gap are nanostructuring graphene into carbon nanotubes (CNTs) and graphene nanoribbons (GNRs) or nanoflakes (GNFs). ${ }^{9,10}$ Many high-quality experimental research efforts have been devoted to understanding the optoelectronic properties of these different topological analogs from graphene, namely GNRs $^{11-14}$ and armchair GNFs of triangular, ${ }^{15}$ hexagonal ${ }^{16,17}$ and other shapes. ${ }^{18,19}$ The scientific community is highly optimistic about the GNR based device fabrication for DNA sequencing along with sophisticated spintronic applications. ${ }^{20-22}$

GNRs are composed of finite-size one-dimensional (1D) straight edges, whereas GNFs are quasi-zero-dimensional graphene quantum dots and are, in principle, part of a large hexagonal carbon network in reduced dimensions. It is important to understand that the building-blocks or the functional components of nanoscale devices should, at the nanometer scale, provide sustainable performance according to Moore's law. It is encouraging to note that, the recent experiments ensure the possibilities of obtaining the graphene features at the scale of tens of nanometers. ${ }^{2}$ Perhaps the quantum confinement attributed to the reduced dimensionality along with the modification of the topological edge-states actuate unique electronic and magnetic properties in the GNFs. The elegant electronic characteristics and contemplation of the application prospects of GNFs have been extensively explored in some recent experimental studies. ${ }^{23-26}$ It is worth mentioning here that the impact of relative variations in size and edge-states on 
the overall electronic structure of relatively large (lateral dimension up to $20 \mathrm{~nm}$ ) GNFs has been experimentally demonstrated. ${ }^{27}$ Localized edge states along with the metallic features are observed in ZZGNFs. The point or extended defect and chemical modification induced magnetism on the non-magnetic graphene crystal is well documented in literature..$^{28-31}$ On the other hand, the non-trivial $\pi$-electron-driven magnetism is the consequence of localized edge-states in a zero-dimensional (0D) graphene nanoflake (GNF). ${ }^{32,33}$ Successful nanofabrication of graphene materials is attributed to contemporary ground-breaking scientific developments, ${ }^{34,35}$ but the controlled growth of a specific edge-type is still challenging. Sophisticated nanofabrication techniques combined with scanning tunneling microscopy produced precise zigzag edges, namely nanoribbons with substantially high long-range magnetic ordering at room temperature. ${ }^{36}$ Interestingly, a transition from semiconductor to metallic state followed by substantial changes to the magnetization of the edgestates, comparable to that of the ribbon width, has also been realized. ${ }^{36}$ The systematic analysis of the conformational changes in the halogen-pi interaction, as depicted by Kim et al., opens-up a new perspective towards size-dependent electronic tuning in the hexagonal carbon network. ${ }^{37}$

It is worth mentioning here that the implicit non-covalent functionalization through the physisorption of electron withdrawing/donating molecules is the implication of a relatively non-destructive and straightforward way to obtain dope graphene. ${ }^{38,39}$ To be precise, the induced doping effect on the graphene surface is the consequence of a charge transfer interaction between the composite pair. Most importantly, the graphene surface institutes minimal deformation due to the predominate van der Waals (vdW) interaction and also maximizes the possibility of the cooperative doping effect. Different experimental and theoretical investigations have been performed to realize the efficient doping of the 2D graphene surface in terms of non-covalent interactions. From the experimental prospects, Voggu et al. successfully carried out p- and n-doping on the graphene surface using specific donor molecules, tetrathiafulvalene (TTF) and some acceptor counterparts like tetracyanoethylene (TCNE). ${ }^{39}$ In another significant contribution, Chen and co-workers designed some doping protocols using the strong acceptor molecule, tetrafluorotetracyanoquinodimethane (F4-TCNQ), to induce p-doping on the surface. ${ }^{38}$ The high-end DFT calculation contributes new insights to this exciting topic. Different groups reported extensive computational simulation results on the non-covalent functionalization of graphene, based on typical electron donors and acceptors such as TTF, ${ }^{40}$ TCNE, $^{41}$ and F4-TCNQ. ${ }^{42}$ Cho et al. performed extensive theoretical investigations to develop a better understanding on the adsorption dynamics of nucleobases on the graphitic surface. ${ }^{43}$ However, none of these reported works realized an opportunity to implement the principle of non-covalent functionalization to account for the size and shape dependent electronic modulations on the zero-dimensional (0D) graphene nanoflakes (GNFs). The non-destructive and reversible nature of functionalization is anticipated to be a pivotal signature to implement the concept in some practical nanoelectronic applications.
In recent years a great deal of research effort has been devoted towards the non-covalent functionalization of graphene. However, to the best of the authors' knowledge, the present study is a systematically organized and compact approach to address a few unexplored aspects of non-covalent interaction arbitrated electronic modulations in graphene nanoflakes. Here, first-principles DFT calculations are used to highlight the acclaimed changes in electronic and magnetic properties of the GNF-HATCN nanocomposites. It is important to note that the electronic environment in quantum dots is mainly sensitive to the shape and size of the system. Therefore, we embodied GNFs constituting different sizes as well as shapes. Subsequently, a strong pi-acceptor molecule, hexaazatriphenylene-hexacarbonitrile (HATCN) (see Fig. S1 in the ESI $\dagger$ ), considerably enhanced the effect of the non-covalent interaction on the GNFs. The six CN groups in the molecule substantially enriched the electron accepting ability of the system. From here onwards we shall use 'HATCN' as the short-hand notation for this molecule.

The paper is organized in the following way: Section 2 contains a brief discussion regarding the implemented computational methodologies along with some relevant information about the model systems. A broad interpretation of the computed data is included in Section 3 and it is sub-divided into two sections. Some of the essential electronic characteristics of the five modeled GNF systems are discussed in Section 3(a). The different electronic and magnetic properties of the donor-acceptor (GNF-HAT) composite systems and their probable futuristic device application prospects are analyzed in Section 3(b). Finally, Section 4 consists of specific concluding remarks on these particular nanocomposites and the possibilities of using non-covalent interactions to regulate the electronic and magnetic properties of nanostructures.

\section{Computational details}

DFT calculations were performed using the open source Quantum Espresso 5.4.0 code. $^{44}$ We used Troullier and Martins ${ }^{45}$ normconserving pseudopotentials and exchange-correlation (xc) effects are incorporated at the Becke-Lee-Yang-Parr (BLYP ${ }^{46}$ level of the generalized gradient approximation (GGA). The van der Waals correction to the calculations has been incorporated through semi-empirical Grimme's DFT-D2 methodology ${ }^{47}$ as implemented in Quantum Espresso. The standard kinetic energy cutoff for wavefunctions is set at 40 Ry and the corresponding cutoff for charge density and potential is set at 160 Ry. As we have discussed previously, the electronic environment of GNFs has a strong dependence on the size and shape of the system. In the present study, we considered five different types of model GNF structures containing zigzag edges. The reciprocal space integrations were performed at the ' $\Gamma$ ' point. Within the extended periodic framework of the calculations, the flakes were considered to be placed in simple cubic supercells such that the periodic images were separated by at least $10 \AA$ and 5-8 $\AA$ vacuum space in the vertical and horizontal directions, respectively, to maintain some negligible interactions between the repetitive units. Subsequently, we have 

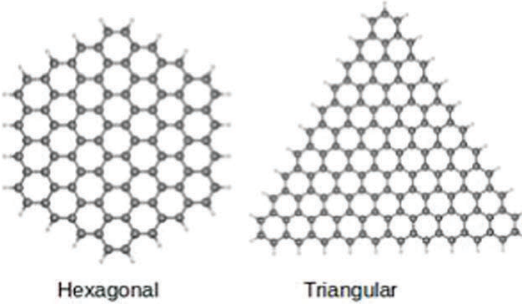

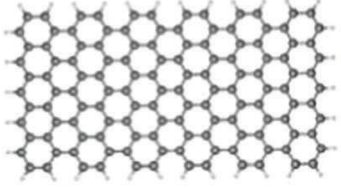

Rectangular

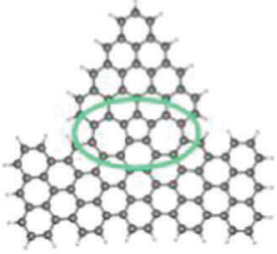

Hybrid

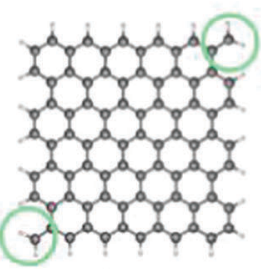

Square

Fig. 1 Relaxed hydrogenated graphene nanoflake (GNF) structures modeled for our present study. We have included different shapes and sizes to account for the topological changes induced electronic modulations in the system. The 'Hybrid' and 'Square' systems contain certain defects in the lattice as depicted by green circles.

performed several convergence tests w.r.t. the different supercell lengths to determine the ground state of the system. Indeed, a $10 \AA$ vacuum space along the $Z$-direction seems to be the most preferred situation to obtain the energy minima for the system. Nanoflakes were optimized until the forces on each atom were less than $5 \times 10^{-3} \mathrm{eV}^{-1}$. The forces on the modeled nanoflakes were minimized up to $<10^{-3}$ Ry a.u. ${ }^{-1}$ for all atoms to obtain fully relaxed atomic geometries. In the model systems, the dangling bonds at the edge atoms are saturated with atomic hydrogens. To understand the impact of shape and size of the GNFs on their electronic environment, we included five different zigzag edge GNF structures (Fig. 1). Furthermore, the topological defects on the GNF are significant to interpret the electronic modulations in the system. To account for this particular feature, we have included two special cases (last two GNF structures in Fig. 1) with existing structural defects. In the composite system, the HATCN molecule is bound to the GNF predominantly through a weak van der Waals type interaction. The acceptor HATCN seems to be strongly physisorbed on the GNF surface. For computational convenience, we have assumed that the GNF is a part of the extended graphene network saturated with atomic hydrogens. We can argue that the $\pi-\pi$ stacking interaction is more favorable for the HATCN-GNF nanocomposite (due to the flat surface structure of both the systems) rather a covalent complex formation. However, we cannot rule out the possibility of covalent complex formation too. In such a situation, the acceptor HATCN will drift to one of the edges of the GNF as electronic charges mostly tend to accumulate on the edges.

\section{Results and discussions}

\section{(a) Impact of topological changes to the electronic and magnetic environment of single layered graphene nanoflakes (GNFs)}

In this section, we will attempt to probe the observed modulations to the electronic environment of GNFs as a result of their topological changes. We have considered five GNF structures with different shapes and sizes along with some defects. The relaxed structures of the model GNFs are given in Fig. 1. It is well established that the intrinsic electronic properties of the GNFs are related to the size and shape of the system. The impact of structural orientation on the electronic charge distribution of five GNFs (from contour plots in the [110] plane) is portrayed in the ESI $\dagger$ (Fig. S2). Some characteristic changes to the symmetric distortion of the electronic charges on the hexagonal and hybrid GNF surfaces are perhaps prevalent in the figure.

It is also essential to shed some light on the edge effect of GNR/GNFs before extending the strategic discussion on the noncovalent interaction mediated electronic modulations. The characteristic charge accumulations on the edges are realized for the GNR based nanostructures. The external transverse electric field induced perturbations to the electronic and magnetic properties of pristine and edge-decorated zGNRs are well documented. ${ }^{48}$ Systematic and controlled chemical modifications to the edges of zGNR lead to an effective decrease in the observed half-metallicity or nonmagnetic behaviors in zGNRs. Some recent scientific developments accounted for the temperature dependence of graphene edge termination at the atomic scale. ${ }^{49}$ This particular experimental study showcased the effect of temperature variations in the edge-state electronic environment of GNRs. At some relatively low temperatures (below $4000{ }^{\circ} \mathrm{C}$ ) the zigzag terminations are prevalent for the graphene edges. On the other hand, the edges are dominated by armchair and reconstructed zigzag edges above $6000{ }^{\circ} \mathrm{C}$.

It is worth mentioning here that the shape and size of the nanoflakes have substantial impact on the intrinsic magnetic properties of the system. The magnetic behavior of the five different systems is reported in Fig. 2. Here, the ground state magnetization densities in terms of up (down) spin densities are represented as red (blue) isosurface plots. Subsequently, the graphical representations of the total and absolute magnetic moment values for the five systems are also included in Fig. 2 . As can be seen in the hexagonal nanoflake, spherical symmetry of the system is preserved and predicted the absence of any magnetic moment for the structure. So, in principle, the hexagonal nanoflake should be non-magnetic in nature. The triangular nanoflake exhibits typical edge-state magnetism. Relatively higher total and absolute magnetic moment values are observed for this structure. The anti-ferromagnetic ordering of the spin arrangement is dominant along the edges.

However, the spin alignment in the triangular GNF is not uniform throughout the edges. It is evident from Fig. 2 that on the contrary to an excess of up-spin densities along the edges, the right-hand corner of the edge experienced sudden growth of excess spin densities with down-spin states. The spin densities 

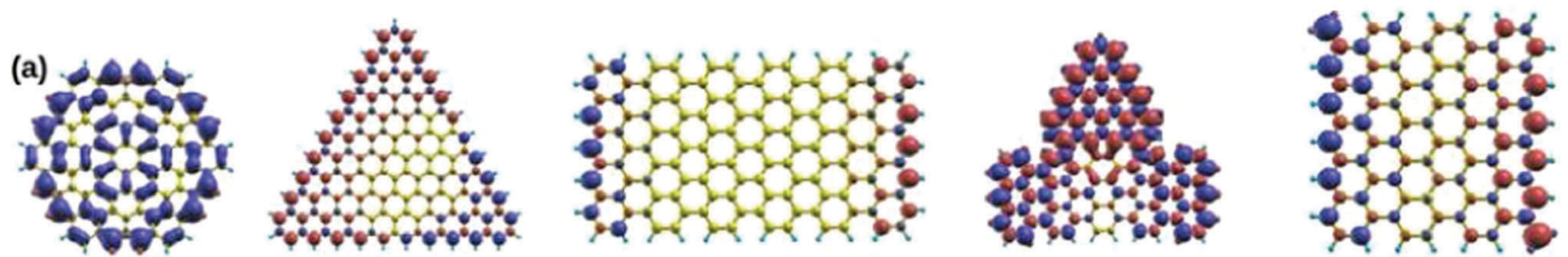

(b)

Magnetization Plot

a total a absolute

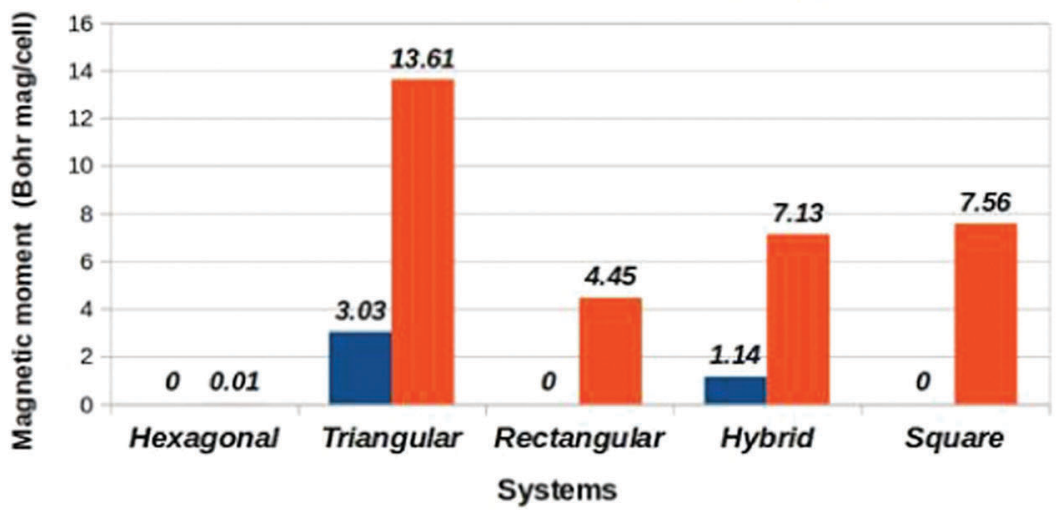

Fig. 2 The magnetic structures of the nanoflake (a) spin density distribution isosurface plots for the five GNFs and (b) corresponding computed total (the integral of the magnetization in the cell) and absolute (the integral of the absolute value of the magnetization in the cell) magnetic moment values. The red and purple color regions in the isosurface plots represent the oppositely aligned excess spin densities.

are localized at the two opposite edges of the rectangular nanoflake. The possibility of anti-ferromagnetic spin coupling between the two opposite edges is distinct in the system. Subsequently, coupling results in zero total magnetic moment in the system, but there are some specific values for the absolute magnetic moment of the system. The hybrid nanoflake attributes specific magnetic moment in the system. The structure is the combination of triangular and rectangular motifs. The magnetic moment in the system is the consequence of the localized spin densities towards the edges of the rectangular fragment. The square fragment also has a significantly low total magnetic moment due to the anti-ferromagnetic interaction between the spin densities located at the two edges. The typical edge-state magnetization is prevalent in all the nanoflake systems.

2D electronic charge density distribution maps for the systems are included in the ESI $\dagger$ (Fig. S2). A uniform electronic charge distribution along the surface of the three nanoflake systems is evident from the figure. However, some anomalous signature has been accounted for in the hexagonal and hybrid systems. Here, the electronic charge localization is observed in some particular regions. The location of the frontier molecular orbitals (FMOs) is an interesting aspect of the GNFs. The computed HOMO-LUMOs are depicted in Fig. 3. Depending on the shape of the GNFs some significant variation in the symmetry of the FMOs has been accounted for, as shown in the figure.
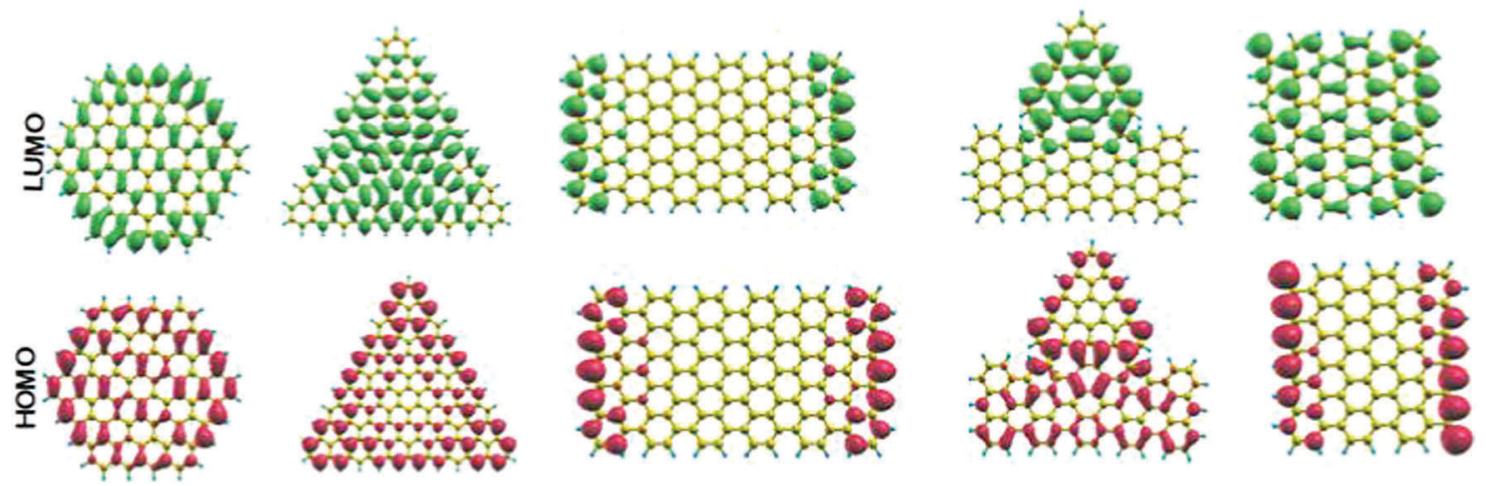

Fig. 3 The highest occupied level (HOMO) and corresponding lowest unoccupied level (LUMO) colored in red (bottom panel) and green (top panel), respectively, for the GNF systems. 
In the hexagonal system, the HOMO and LUMO are symmetric in nature. The lobes are symmetrically spread along the surface. The HOMO in the triangular GNF is localized more towards the edges while the LUMO is squeezed to the center of the system. In the rectangular system, the FMOs are concentrated at the two opposite edges. The hybrid system has some different symmetric orientations for the FMOs, as the HOMO is mainly located on the rectangular part whereas the triangular portion of the structure hosts the LUMO. Similarly, for the square GNF, the HOMO contributions have appeared along the edges, and the LUMO is distributed across the whole structure.

\section{(b) Non-covalent interaction induced modulations to the electronic environment}

In this section we shall systematically evaluate the possibilities of non-bonding interactions as effective external stimuli to tune the electronic properties of graphene nanoflakes (GNFs). As we know, the GNF surface has some inherent electron donor characteristics, so a strong electron acceptor is imperative to induce electronic modulation to the system. The overall system will behave as a composite donor-acceptor nanostructure. Subsequently, the explicit charge transfer between the two components effectively regulates the electronic environment of the GNF. Indeed, the non-covalent functionalization with organic molecules accounts for reversible electronic perturbations on the graphene. Perhaps the weak van der Waals type interactions between the $\pi$-orbitals of the planar organic molecule and graphene are the origin of observed electronic changes in the system. Recently, this simple functionalization technique has been exploited to modify the electronic properties of a graphene surface with different organic molecules such as tetrafluorotetracyanoquinodimethane (F4-TCNQ), ${ }^{50}$ 3,4,9,10-perylenetetracarboxylic dianhydride (PTCDA), ${ }^{51}$ etc. In search of a suitable surface modifier, hexaazatriphenylene hexacarbonitrile (HATCN) turns out to be an ideal candidate. Some recent computational studies on HATCN/graphene accounted for the significant work function $(\Phi)$ modification along with the persistence of large charge carrier mobilities in the resulting doped graphene surface. ${ }^{52}$ Also, this molecule has a considerable electron affinity (EA) that pushes the lowest unoccupied molecular orbital (LUMO) level close to the Fermi level of an electrode. ${ }^{53}$ The electronic properties of HATCN are well documented in the literature. ${ }^{54,55}$
The zero-dimensional GNFs appeared to have a flat surface similar to that of graphene with the characteristic edge-states. To assess the electronic fingerprint of non-covalent functionalization on the GNFs, the HATCN molecule is placed horizontally on top of the GNFs in our model systems. We have systematically screened a number of different initial structural conformations of HATCN adsorbed on the GNF surface. It is worth mentioning here that the observed relative energy difference is found to be negligible for the different conformations. Out of the tested orientations, our current study will be concentrated on the most stable configurations. The relaxed geometries of the systems are reported in Fig. 4. In the subsequent sections, we will elaborate the comprehensive understandings on the relative changes to the electronic environment of different GNFs by the non-covalent functionalization with HATCN.

(I) Structural stability of the systems. The primary component of the stabilization in the composite systems is the non-covalent interactions, mainly induced from van der Waals type forces. HATCN exhibits strong electrostatic interaction of the $\mathrm{CN}$ groups in addition to the sizeable $\pi-\pi$ interaction with the conjugated core. The resulting interaction energies of the systems are predicted to be sufficient enough to impart thermodynamic stability to the combined nanostructure. The pictorial representation of the interaction energy plot is given in Fig. 5.

As observed from the interaction energy plots, the size and shape of the GNFs proclaimed distinct variations in the overall stability of the nanostructures. The nanoflake systems with hexagonal (DCC) and hybrid structures are predicted to have relatively lower stability with HATCN. Again, the non-covalent interaction between HATCN and the rectangular GNF nanocomposite institutes the highest stability among the considered systems. Similarly, the structures with square and triangular shaped GNFs and HATCN showed substantial improvements in their thermodynamic stabilities compared to those of the hexagonal and hybrid systems. It can be realized that depending on the size and shape of the GNFs there are some significant changes to the overall stability of the combined systems. Predominantly, dispersion interactions between the HATCN and GNF holds the two fragments and an associated charge transfer provides the additional increment to the overall stability.
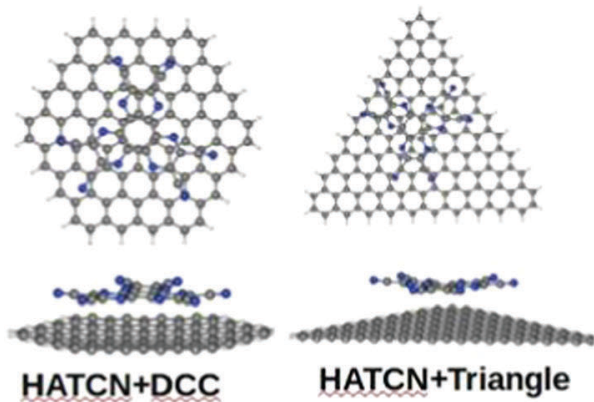

HATCN+Triangle
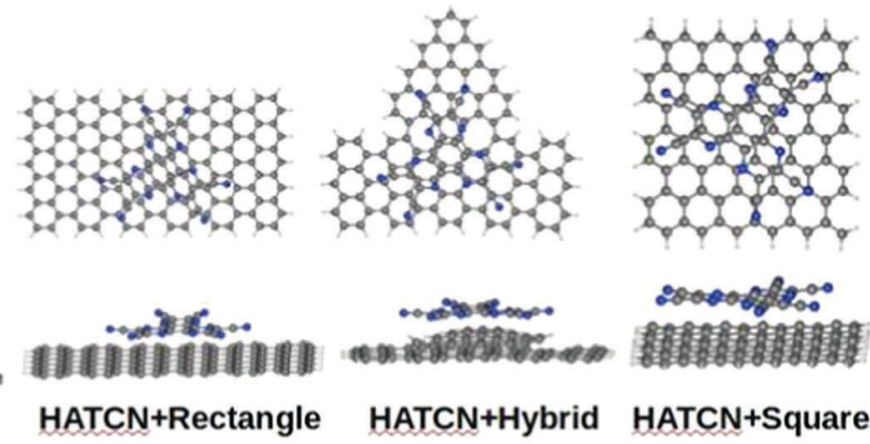

HATCN+Hybrid

HATCN+Square

Fig. 4 The relaxed structures of the GNF-HATCN composite system. The top is the vertical view of the system, and below is the horizontal view. 


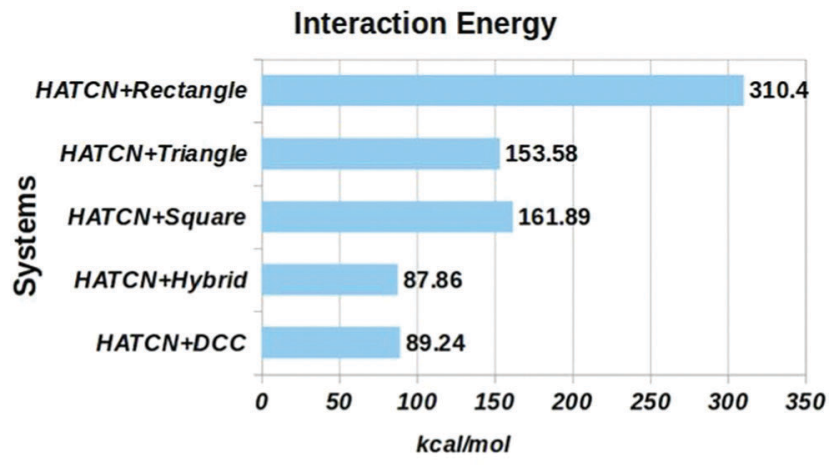

Fig. 5 The interaction energy plots for the five GNF-HATCN composite systems. The energy value is calculated as Interaction Energy $=E_{\mathrm{GNF}-\mathrm{HATCN}}-$ $\left(E_{\mathrm{GNF}}+E_{\mathrm{HATCN}}\right)$.

(II) Electronic structure. A better understanding of the electronic environment of these particular HATCN-GNF nanocomposites is necessary to realize the application prospects of the model structures. Here we shall present some useful insights into the predicted electronic behavior of the system. It is well understood that the unusual electronic properties of systems at the nanometer scale primarily originate from the electron confinement effect. It is observed that structural (shape and size) changes to the nanostructure can smoothly modulate the overall electronic properties of the system. The effective structural changes broadcast the possibilities of tuning the electronic environment with some external stimuli. It is worth mentioning here that the HATCN molecule acts as the customized molecular switch to regulate electronic tuning. The density of states (DOS) spectrum for the systems is reported in Fig. S3 (in the ESI $\dagger$ ). Distinct variations in the electronic structure of the double-layered composite systems are evident from the computed DOS spectrum figure. Here we stressed the electronic modulations induced from the through-space interaction between the GNF and HATCN. The spin-resolved DOS exhibits highly intense peaks just below and above the Fermi level. Indeed, these sharp peaks are accounted for primarily by the edge-state pi-electron contributions.

The resulting changes in the electronic states predominantly arise from the variation in size and shape of the GNFs. The symmetric distribution of the electronic energy states for the up and down spin electrons is prevalent in the hexagonal-HATCN system. A fully compensated ferrimagnetic ordering in the system is also accounted for in these symmetric DOS patterns. The intense peaks at the Fermi level indicate the possibility of intermolecular charge transfer in the system. Interestingly, some half-metallic conducting nature of the electrons is evident for the triangular-HATCN system. Here, electrons in the up-spin channel show metallic properties while the down-spin electrons seem to have an insulating nature. The rectangular flake remained half-metallic but alters electronic energy states at the Fermi level. As can be seen from the figure that the downspin electron channels located below the Fermi level are associated with metallic behavior. The nanocomposite consisting of the hybrid GNF exhibits metallic characteristics. The ferromagnetic spin arrangement in the system can also be realized from the DOS spectrum. The electronic structure in the square-HATCN system is qualitatively similar to that of the triangular GNF composite. The up-spin channels are populated at the Fermi level and there is a substantial energy gap between the highest occupied and lowest unoccupied states in the down-spin channels. This represents the ideal half-metallic nature of the system. The majority of the systems considered
Projected Density of States (PDOS)

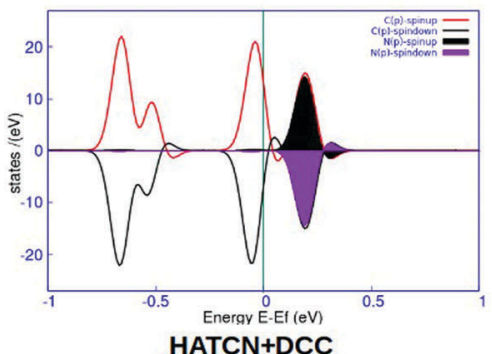

Projected Density of States (PDOS)

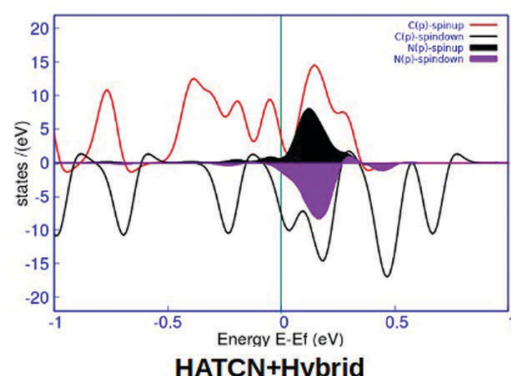

HATCN+Hybrid
Projected Density of States (PDOS)

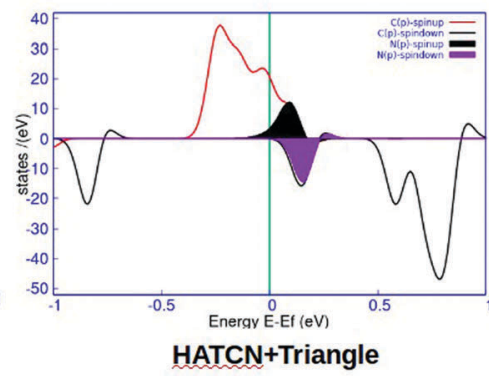

Projected Density of States (PDOS)

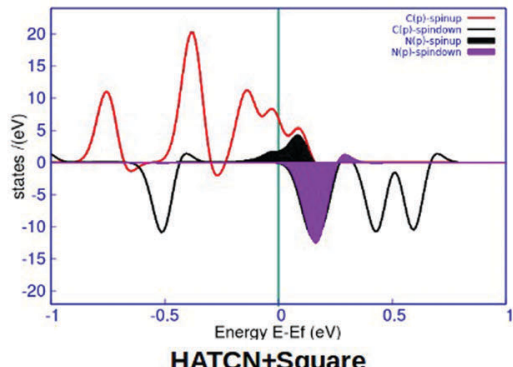

HATCN+Square
Projected Density of States (PDOS)

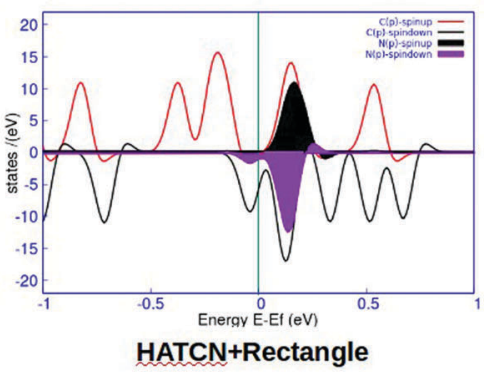

Fig. 6 The projected density of states (PDOS) plots for the five different GNF-HATCN nanosystems. Here, the Fermi level is set at zero. 
in the present study remain half-metallic upon interaction with HATCN. Perhaps these hypothetical systems anticipated manifesting comprehensive spin-polarized transport under certain gate voltage. Furthermore, the findings pertained certain control over the electronic conduction in a particular channel by tuning the polarity of the gate voltage in the composite structures. It is apparent that the structural features of the GNFs along with external stimuli, such as HATCN, are effective tools to regulate spin-polarized transport in such systems.

The relative contributions of different atoms present in the system toward the overall electronic structure provide a comprehensive understanding of the electronic transport properties of the nanocomposite. Subsequently, Fig. 6 depicts the spinpolarized partial density of states (PDOS) plot for the different systems. Here, red and black lines represent the density of spin up and down p-electrons of carbon atoms, whereas the filled regions (inside the plots) are the contributions from the p-electrons of the nitrogen atoms (spin up and down) present in HATCN. The substantial contributions from the nitrogen p-electrons at the Fermi level are evident from the figure. It can also be argued that the inherent half-metallic nature of the systems is mainly influenced by the presence of the nitrogen atoms. It is observed that the electronic states of the nitrogen atoms are the solo contributors to the down-spin channels at the Fermi level to impart half-metallicity to the system. This institutes some persuasive indication that the non-covalent interaction induced tuning of electronic properties in GNF probably has some vital importance in the context of spin-based electronics.

The propagations of an electronic wavefunction at the valence band maxima (VBM) and conduction band minima (CBM) are reported in Fig. 7. The VBM is concentrated on the
GNFs, and the CBM is primarily located on the acceptor part of the composite system. This indicates higher probabilities for the through-space charge transfer between the two fragments. In the hexagonal system, the VBM is symmetrically distributed throughout the GNF sheet, and the CBM is positioned at the HATCN. On the other hand, the VBM is mostly concentrated on one of the edges of the GNF in the triangular system, and some asymmetric isosurface densities for the CBM are accounted for in the figure. The rectangular system exhibited well-separated edge-state VBMs, positioned at the GNF followed by the presence of symmetric CBM state identities at the HATCN. In the hybrid GNF-HATCN composite, the VBM is positioned at the triangular part of the system, while the CBM is primarily concentrated at the HATCN. We have observed some distinct changes in the distribution of FMOs for the square-shaped GNF system. Here, both the VBM and CBM wavefunctions are located on the GNF sheet with different symmetries. It is worth mentioning here that we have observed some substantial changes in the HOMO-LUMO gap values for the systems. The graphical representation of the HOMO-LUMO gap values is reported in Fig. 8. It is quite convincing that the shape of the GNF system has significant impact on the overall HOMO-LUMO gap of the system. As we have seen (Fig. 8), the hybrid-HATCN and triangle-HATCN composite systems have the lowest and highest gap values, respectively.

The important aspect of the present study is the fabrication of non-covalent interaction mediated stable donor-acceptor nanocomposites and understanding the possible tuning of the electronic properties of the systems. The electronic structure, as well as the chemical nature, of a system is primarily dependent on the properties of frontier molecular orbitals.
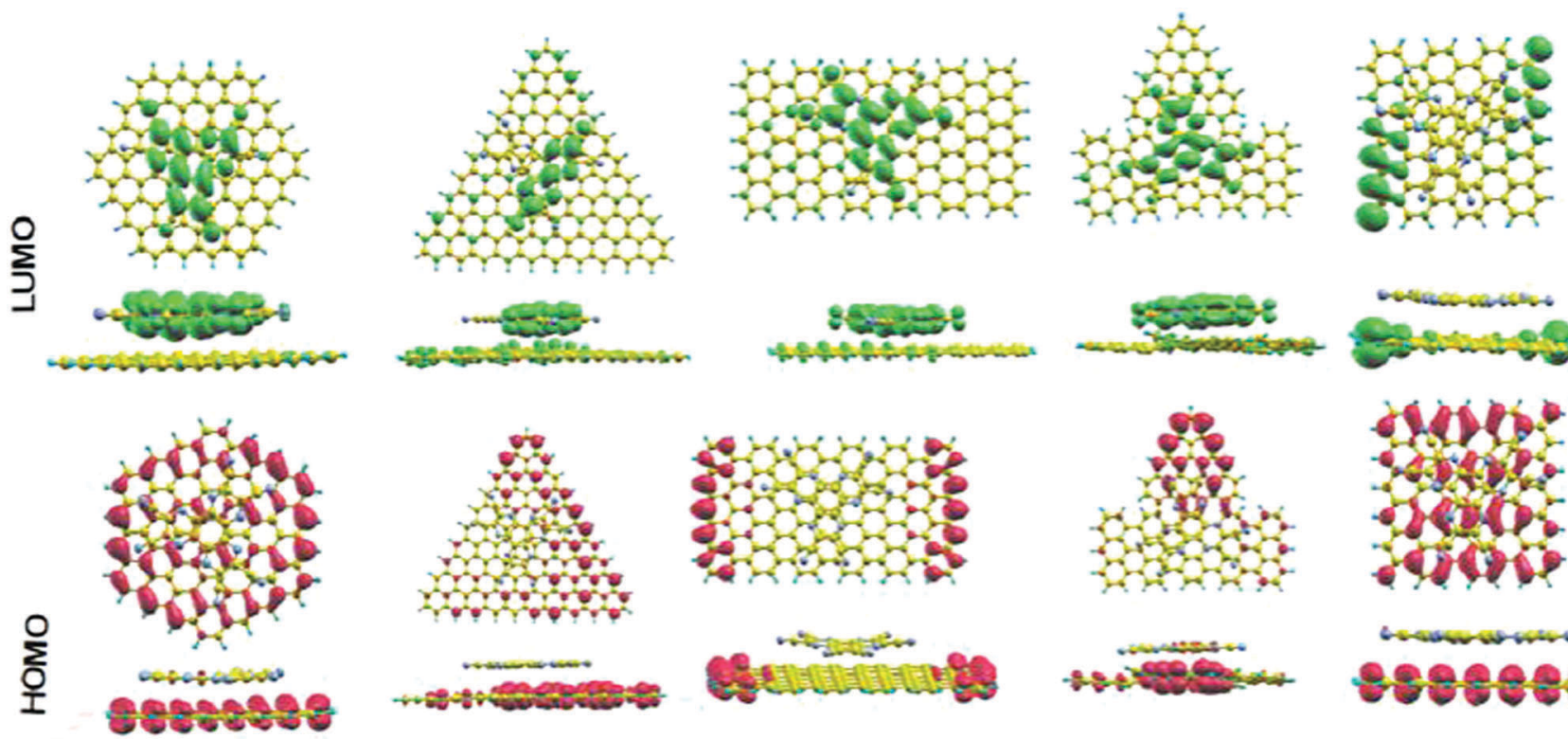

Hexagonal+HATCN
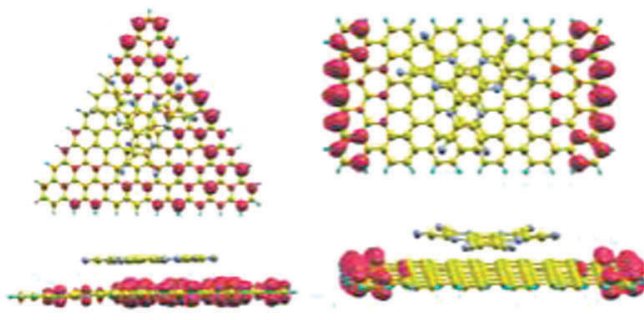

Triangular+HATCN
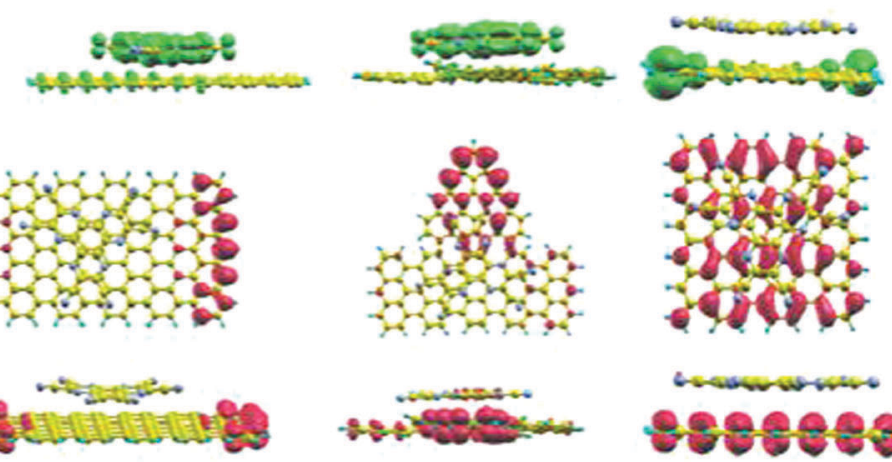

Hybrid+HATCN

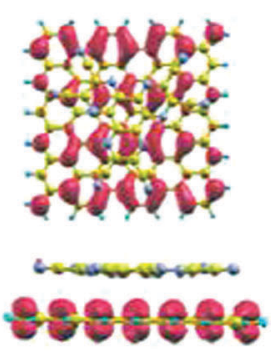

Square $+\mathrm{HATCN}$

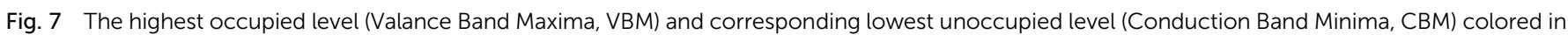
red (bottom panel) and green (top panel), respectively, for the systems. 


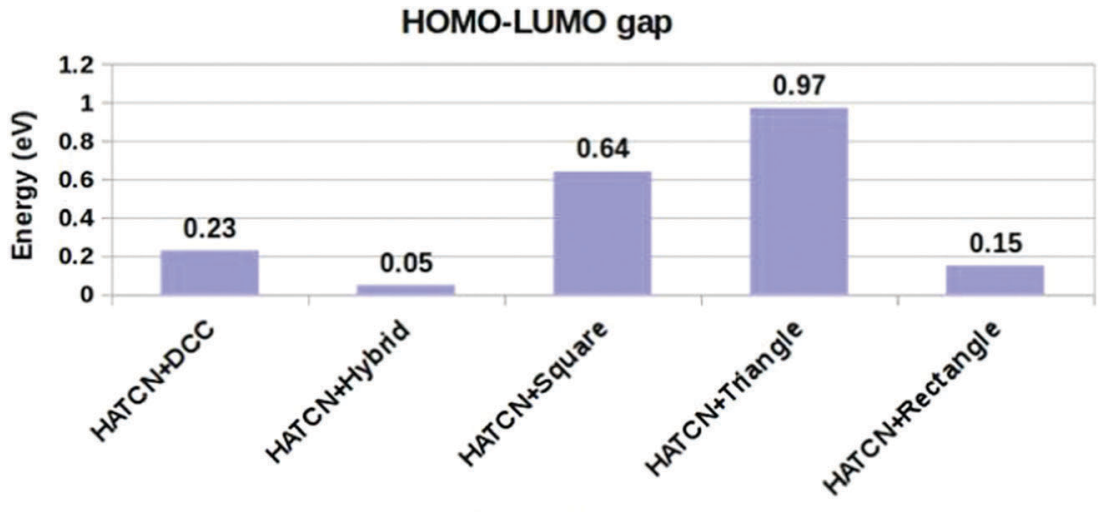

Systems

Fig. 8 Graphical representations of the computed HOMO-LUMO gap values for the five systems in ascending order.

As we have discussed, the qualitative interpretations of the computed FMO pictures along with the relative HOMO-LUMO gap values provide certain strategic realizations to our concept. Perhaps there are two parameters that control the electronic changes in the system. To accomplish this task, we kept the acceptor (HATCN) fixed and varied the donor (GNFs) to regulate the desirable changes to the electronic properties of the combined system. It is worth mentioning here that the non-covalent interaction of the GNFs with a strong acceptor develops some conceptual understandings on the promising aspect of non-destructive and reversible functionalization of carbon quantum dots.

We have discussed the magnetic properties of single layer GNFs in the previous sections. Subsequently, some distinct changes to the original spin-arrangement have been observed for the GNFs upon the incorporation of HATCN. The computed 3D isosurface spin density maps are depicted in Fig. 9. Inevitably, the non-covalent interactions trigger observable modulations to the spin-dependent electronic properties of the GNFs. A pictorial representation of the total magnetic moment values for the composite systems is included in the ESI $\dagger$ (Fig. S4). The symmetric spin arrangement in the hexagonal system does not have a noticeable impact due to the introduction of HATCN, and the spin distribution almost remains the same as that of the isolated GNF.
However, HATCN imparted substantial changes to the spinarrangement in the triangular GNF system. We have observed the effect of spin reorganization in the spin density distribution upon placing HATCN above the triangular GNF surface. The growth of opposite spin densities at one of the edges of the isolated triangular GNF system has been suppressed, and the typical edge-state anti-ferromagnetic spin arrangement is consistent along the edges of the nanostructure. Similar observations are also accounted for in the rectangular GNF. Antiferromagnetically coupled edge-state magnetism is prevalent in the isolated rectangular GNF system, but the incorporation of HATCN on top of the GNF activates the development of strong ferromagnetically coupled states between the opposite edges. The non-covalent interaction between the systems regulates the observed spin transition. The hybrid GNF also shows changes to the spin orientation in the presence of HATCN. The excess spin density growth on the HATCN surface is prevalent in the composite structure. The typical anti-ferromagnetic spin arrangement is prominent at the triangular portion of the system followed by a substantial decrease in spin densities on the rectangular region. The spin rearrangement effect is also observed in the square GNF and HATCN system. As we have seen the particular anti-ferromagnetic spin orientations in the two opposite edges is predominant in the isolated GNF. Subsequently, the non-covalent

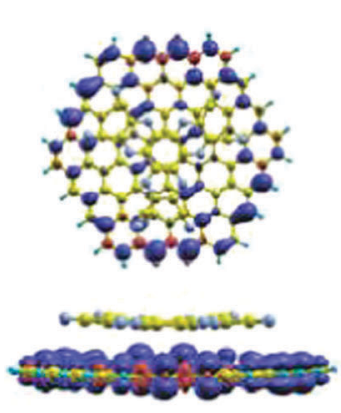

Hexagonal+HATCN
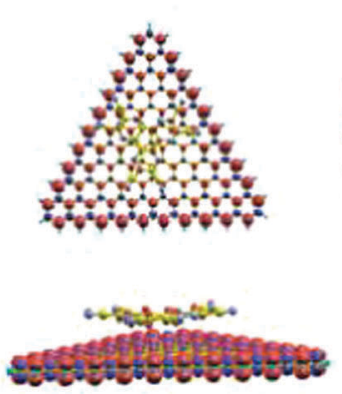

Triangular+HATCN
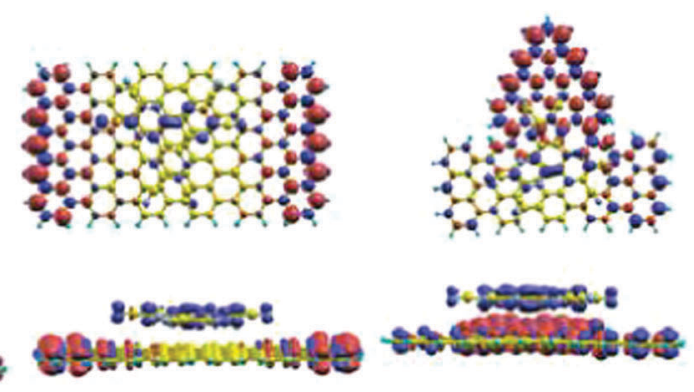

Hybrid+HATCN

Rectangular+HATCN
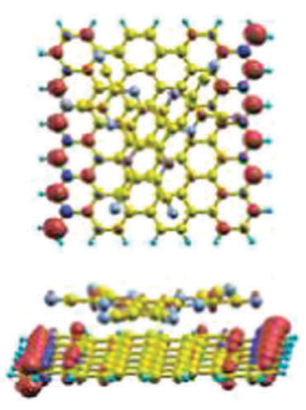

Square+HATCN

Fig. 9 The isosurface spin density distribution plots for the five different GNF-HATCN nanocomposites. The red and purple colored regions in the plot represent the oppositely aligned excess spin densities. 
as well as charge transfer interactions between the two fragments lead to the formation of ferromagnetically coupled edge-state spin orientations. The graphical pattern of the total magnetization is similar to that of the HOMO-LUMO gaps reported in the study. As we have emphasized, the non-covalent interaction mediated modulations to the spin states is also a reversible process. Subsequent removal of the external stimuli restored the original magnetic behavior of the GNF system. The induced changes are not permanent, and the effective modulations can be turned on/off by inclusion of the donor fragment. Indeed, the shape and size of the GNF is a vital tuning parameter to synchronize the changes.

(III) Interfacial charge transfer. In order to develop core understandings on the interfacial electronic properties, we have computed the planar average charge density difference, $\Delta \rho(z)$, for different composite structures, which is given as

$$
\Delta \rho(z)=\rho(z) \text { combined }-(\rho(z) \mathrm{GNF}+\rho(z) \text { HATCN })
$$

Here, $\rho(z)$ combined, $\rho(z) \mathrm{GNF}$, and $\rho(z)$ HATCN are the planar average charge densities of the combined, GNF, and HATCN layers, respectively, i.e. charge density integrated along the $x-y$ plane (surface area) of the supercell and given only along the $z$-direction, where

$$
\rho(z)=\int \rho(x, y, z) \mathrm{d} x \mathrm{~d} y
$$

Physically, $\Delta \rho(z)$ is a quantitative measure of the charge reorganization in the combined system due to the molecular adsorption. The graphical representations of the computed $\Delta \rho(z)$ values are corroborated in Fig. 10 . The individual positions of the systems are depicted along the $x$-axis and the corresponding charge reorganizations are plotted along the $y$-axis. The negative (positive) values in $\Delta \rho(z)$ signify the adsorption-induced electron density depletion (accumulation) patterns in the process. The pictorial representations of the 3D isosurface density difference maps are also included in the ESI $\dagger$ (Fig. S5). These isosurface maps provide some more profound insights into the electronic charge transfer and reorganization equilibrium associated with the formation of the nanocomposite. The importance of charge reorganization before and after the charge transfer between the different components of the overall structure is also evident from the isosurface maps. In Fig. 10 the black dotted line distinguishes the interfacial position between GNF and HATCN in the supercell. A significantly high amount of electronic reorganization on the GNF surface (represented by the two sharp peaks on the left) is the characteristic feature in all the systems. The charge reorganization has been followed by the sizeable interfacial charge transfer between the GNF and HATCN (the two adjacent up and down peaks near the dotted line). The only exception is the hybrid GNF and HATCN system, where the interfacial charge transfer has been restricted due to the higher degrees of charge reorganization, as evident from the plot. Finally, the accumulated charges on HATCN undergo certain degrees of reorganization to settle down
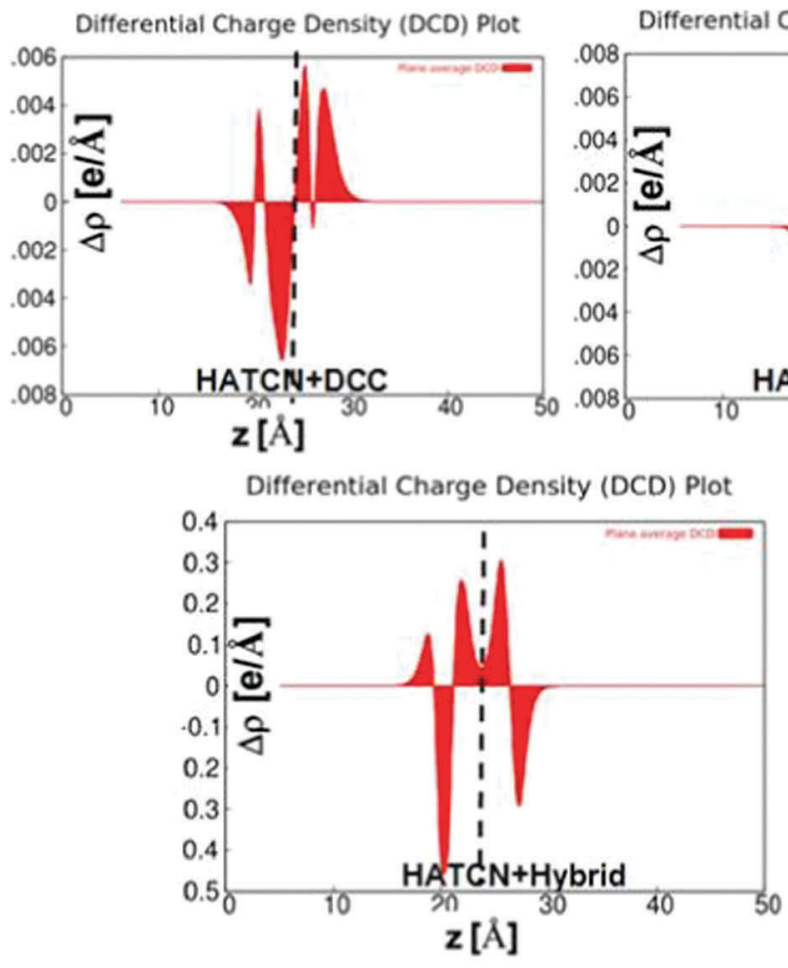
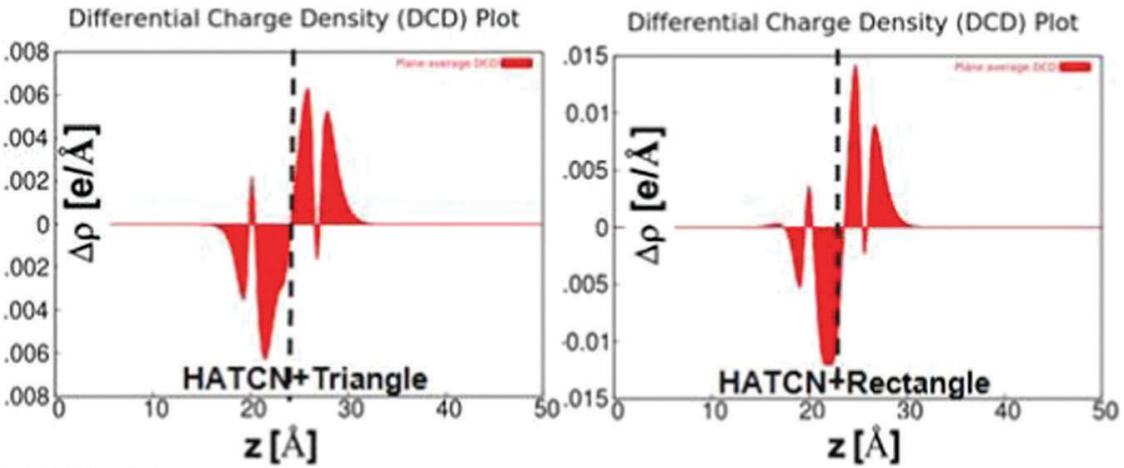

Differential Charge Density (DCD) Plot

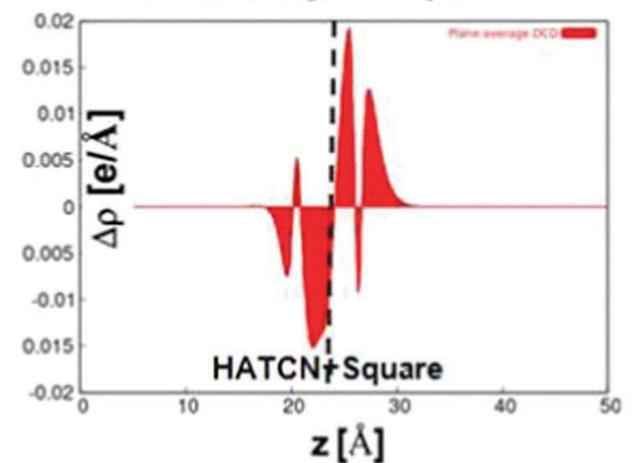

Fig. 10 The planar average differential charge density (DCD), $\Delta \rho(z)$, plots for the HATCN-GNF nanocomposite systems, w.r.t the distance along the $z$-axis $z\left[A^{\prime}\right]$. The black dotted line highlights the interfacial position. Here, the positive and negative values provide some quantitative approximations to the electron depletion and accumulation, respectively. 
(last two peaks at the right end). This is a qualitative analysis to understand the charge transfer prospect in the system. The representative DCD isosurface density maps, provided in the ESI $\dagger$, are also in reasonable agreement to the plots. The electron depletion and accumulation zones are represented by the red and blue colors, respectively. The charge transfer pattern is almost similar in all the systems. However, depending on the shape of the GNF the charge reorganization is bit unclear and provides scope for some comprehensive investigation to develop a better understanding.

\section{Conclusions}

We have investigated the emergence of non-covalent interaction mediated modulations on the electronic properties of graphene nanoflakes. The experimental realization for controlled preparation of GNFs with a wide range of size variation (ranging from several nanometers to a few hundred nanometers) already exists. ${ }^{56}$ Our DFT calculations suggest that controlled perturbations can be introduced to the electronic structure of GNFs through noncovalent interactions with strong $\pi$-acceptor systems. Essentially, the observed electronic changes are temporary and are effectively turned-off by removing the external stimuli. Our present study also shed some light on the unconventional functionalization of zerodimensional GNF using a non-destructive pathway. The electronic behavior of these donor-acceptor composites is analyzed by different computed parameters. Eventually the shape and size dependent critical changes to the electronic properties of the systems are also systematically evaluated. It is important to note that the particular shape of the GNF is crucial to pertaining the desirable tuning of the electronic properties of the composite system. As we have discussed, the dispersion and charge transfer interactions complement each other to impart overall stability to the system. The interfacial charge transfer regulates the observed electronic changes in the composite structure. Considerable modulations to the spin-dependent properties of the system as a result of non-covalent interactions are also accounted for in the present study. The non-covalently attached acceptor can effectively reorganize the typical spin-arrangements in the GNFs. Consequently, there are some distinct changes to the magnetic behavior of the system. Moreover, the anti-ferromagnetic to ferromagnetic switching in the edge-state magnetism of GNF is an important perspective of our current study. These findings institute realistic possibilities of the self-regulated device fabrications for such composite nanostructures. Indeed, nanometersized GNFs possess the potential advantage to become buildingblocks in nanoscale electronics. Additionally, a comprehensive understanding of the electronic behavior of GNFs along with the tools to induce controlled modulations opens-up new avenues for future nanoscale device fabrication.

\section{Conflicts of interest}

There are no conflicts of interest to declare.

\section{Acknowledgements}

This work was supported by the research project RVO 61388963 of the Czech Academy of Sciences. We acknowledge the financial support of the Czech Science Foundation (AS, PH: P208/12/G016). This work was supported by the Ministry of Education, Youth and Sports from the Large Infrastructures for Research, Experimental Development and Innovations project "IT4Innovations National Supercomputing Center - LM2015070" as well as project LO1305 (PH).

\section{References}

1 T. L. Makarova, Semiconductors, 2004, 38, 615-638.

2 A. K. Geim and K. S. Novoselov, Nat. Mater., 2007, 6, 183-191.

3 K. Kawasumi, Q. Zhang, Y. Segawa, L. T. Scott and K. Itami, Nat. Chem., 2013, 5, 739-744.

4 K. S. Novoselov, A. K. Geim, S. V. Morozov, D. Jiang, Y. Zhang, S. V. Dubonos, I. V. Grigorieva and A. A. Firsov, Science, 2004, 306, 666-669.

5 J. C. Meyer, A. K. Geim, M. I. Katsnelson, K. S. Novoselov, T. J. Booth and S. Roth, Nature, 2007, 446, 60-63.

6 K. S. Novoselov, Z. Jiang, Y. Zhang, S. V. Morozov, H. L. Stormer, U. Zeitler, J. C. Maan, G. S. Boebinger, P. Kim and A. K. Geim, Science, 2007, 315, 1379.

7 Y. W. Son, M. L. Cohen and S. G. Louie, Nature, 2006, 444, 347-349.

8 K. S. Novoselov, A. K. Geim, S. V. Morozov, D. Jiang, M. I. Katsnelson, I. V. Grigorieva, S. V. Dubonos and A. A. Firsov, Nature, 2005, 438, 197-200.

9 W. L. Ma and S. S. Li, Appl. Phys. Lett., 2012, 100, 163109.

10 S. Kilina and S. Tretiak, Adv. Funct. Mater., 2007, 17, 3405-3420.

11 X. Li, X. Wang, L. Zhang, S. Lee and H. Dai, Science, 2008, 319, 1229-1232.

12 L. Jiao, L. Zhang, X. Wang, G. Diankov and H. Dai, Nature, 2009, 458, 877-880.

13 D. V. Kosynkin, A. L. Higginbotham, A. Sinitskii, J. R. Lomeda, A. Dimiev, B. K. Price and J. M. Tour, Nature, 2009, 458, 872-876.

14 J. Cai, P. Ruffieux, R. Jaafar, M. Bieri, T. Braun, S. Blankenburg, M. Muoth, A. Seitsonen, M. Saleh, X. Feng, K. Müllen and R. Fasel, Nature, 2010, 466, 470-473.

15 M. Treier, C. A. Pignedoli, T. Laino, R. Rieger, K. Müllen, D. Passerone and R. Fasel, Nat. Chem., 2011, 3, 61-67.

16 F. Doetz, J. D. Brand, S. Ito, L. Gherghel and K. Muellen, J. Am. Chem. Soc., 2000, 122, 7707-7717.

17 L. Zhi and K. Müllen, J. Mater. Chem., 2008, 18, 1472-1484.

18 R. Liu, D. Wu, X. Feng and K. Müllen, J. Am. Chem. Soc., 2011, 133, 15221-15223.

19 I. Snook and A. Barnar, in Physics and Applications of Graphene - Theory, ed. S. Mikhailov, InTech, 2011, ISBN 978-953-307-152-7.

20 S. K. Min, W. Y. Kim, Y. Cho and K. S. Kim, Nat. Nanotechnol., 2011, 6, 162-165.

21 Y. Kim and K. S. Kim, Nat. Nanotechnol., 2008, 3, 408-412. 
22 A. C. Rajan, M. R. Rezapour, J. Yun, Y. Cho, W. J. Cho, S. K. Min, G. Lee and K. S. Kim, ACS Nano, 2014, 8, 1827-1833.

23 E. Kan, W. Hu, C. Xiao, R. Lu, K. Deng, J. Yang and H. Su, J. Am. Chem. Soc., 2008, 130, 4224-4225.

24 Y. Zhou, Z. Wang, P. Yang, X. Sun, X. Zu and F. Gao, J. Phys. Chem. C, 2012, 116, 5531-5537.

25 S. Kim, S. W. Hwang, M. K. Kim, D. Y. Shin, D. H. Shin, C. O. Kim, S. B. Yang, J. H. Park, E. Hwang, S. H. Choi, G. Ko, S. Sim, C. Sone, H. J. Choi, S. Bae and B. H. Hong, ACS Nano, 2012, 6, 8203-8208.

26 S. H. Jin, D. H. Kim, G. H. Jun, S. H. Hong and S. Jeon, ACS Nano, 2013, 7, 1239-1245.

27 K. A. Ritter and J. W. Lyding, Nat. Mater., 2009, 8, 235-242.

28 O. V. Yazyev and L. Helm, Phys. Rev. B: Condens. Matter Mater. Phys., 2007, 75, 125408.

29 J. J. Palacios, J. Fernández-Rossier and L. Brey, Phys. Rev. B: Condens. Matter Mater. Phys., 2008, 77, 195428.

30 S. S. Alexandre, A. D. Lucio, A. H. C. Neto and R. W. Nunes, Nano Lett., 2012, 12, 5097-5102.

31 H. González-Herrero, J. M. Gómez-Rodríguez, P. Mallet, M. Moaied, J. J. Palacios, C. Salgado, M. M. Ugeda, J. Y. Veuillen, F. Yndurain and I. Brihuega, Science, 2016, 352, 437-441.

32 M. Fujita, K. Wakabayashi, K. Nakada and K. Kusakabe, J. Phys. Soc. Jpn., 1996, 65, 1920-1923.

33 Z. Klusek, Z. Waqar, E. Denisov, T. Kompaniets, I. Makarenko, A. Titkov and A. Bhatti, Appl. Surf. Sci., 2000, 161, 508-514.

34 N. Tombros, C. Jozsa, M. Popinciuc, H. T. Jonkman and B. J. van Wees, Nature, 2007, 448, 571-574.

35 M. Y. Han, B. Özyilmaz, Y. Zhang and P. Kim, Phys. Rev. Lett., 2007, 98, 206805.

36 G. Z. Magda, X. Jin, I. Hagymasi, P. Vancso, Z. Osvath, P. Nemes-Incze, C. Hwang, L. P. Biro and L. Tapaszto, Nature, 2014, 514, 608-611.

37 D. Y. Kim, J. M. L. Madridejos, M. Ha, J. H. Kim, D. C. M. Yang, C. Baig and K. S. Kim, Chem. Commun., 2017, 53, 6140-6143.

38 W. Chen, S. Chen, D. C. Qi, X. Y. Gao and A. T. S. Wee, J. Am. Chem. Soc., 2007, 129, 10418-10422.
39 R. Voggu, B. Das, S. C. Rout and C. N. R. Rao, J. Phys.: Condens. Matter, 2008, 20, 472204.

40 J. T. Sun, Y. H. Lu, W. Chen, Y. P. Feng and A. T. S. Wee, Phys. Rev. B: Condens. Matter Mater. Phys., 2010, 81, 155403.

41 A. K. Manna and S. K. Pati, Chem. - Asian J., 2009, 4, 855-860.

42 X. Q. Tian, J. B. Xu and X. M. Wang, J. Phys. Chem. B, 2010, 114, 11377-11381.

43 Y. Cho, S. K. Min, J. Yun, W. Y. Kim, A. Tkatchenko and K. S. Kim, J. Chem. Theory Comput., 2013, 9, 2090-2096.

44 P. Giannozzi, et al., J. Phys.: Condens. Matter, 2009, 21, 395502.

45 N. Troullier and J. L. Martins, Phys. Rev. B: Condens. Matter Mater. Phys., 1991, 43, 1993.

46 J. P. Perdew, K. Burke and M. Ernzerhof, Phys. Rev. Lett., 1996, 77, 3865-3868.

47 S. Grimme, J. Comput. Chem., 2006, 27, 1787-1799.

48 M. R. Rezapour, J. Yun, G. Lee and K. S. Kim, J. Phys. Chem. Lett., 2016, 7, 5049-5055.

49 C. Hyun, J. Yun, W. J. Cho, C. W. Myung, J. Park, G. Lee, Z. Lee, K. Kim and K. S. Kim, ACS Nano, 2015, 9, 4669-4674.

50 H. Huang, S. Chen, X. Gao, W. Chen and A. T. S. Wee, ACS Nano, 2009, 3, 3431-3436.

51 Y. Y. Choi, S. J. Kang, H. K. Kim, W. M. Choi and S. I. Na, Sol. Energy Mater. Sol. Cells, 2012, 96, 281-285.

52 C. Christodoulou, A. Giannakopoulos, M. V. Nardi, G. Ligorio, M. Oehzelt, L. Chen, L. Pasquali, M. Timpel, A. Giglia and S. Nannarone, et al., J. Phys. Chem. C, 2014, 118, 4784-4790.

53 L. Zhang, F. S. Zu, L. I. Deng, F. Igbari, Z. K. Wang and L. S. Liao, ACS Appl. Mater. Interfaces, 2015, 7, 11965-11971.

54 L. Chen, L. Wang, Z. Shuai and D. Beljonne, J. Phys. Chem. Lett., 2013, 4, 2158-2165.

55 J. Hwang, H. K. Choi, J. Moon, T. Y. Kim, J. W. Shin, C. W. Joo, J. H. Han, D. H. Cho, J. W. Huh and S. Y. Choi, et al., Appl. Phys. Lett., 2012, 100, 133304.

56 P. Leicht, L. Zielke, S. Bouvron, R. Moroni, E. Voloshina, L. Hammerschmidt, Y. S. Dedkov and M. Fonin, ACS Nano, 2014, 8, 3735-3742. 